\title{
Quasigeostrophic Diagnosis of Mixed Layer Dynamics Embedded in a Mesoscale Turbulent Field
}

\author{
CÉDRIC P. CHAVANNE \\ Institut des Sciences de la Mer de Rimouski, Université du Québec à Rimouski, Rimouski, Quebec, Canada \\ PATRICE KLEIN \\ Laboratoire de Physique des Océans, IFREMER-CNRS-UBO-IRD, Plouzané, France
}

(Manuscript received 28 August 2014, in final form 3 November 2015)

\begin{abstract}
A quasigeostrophic model is developed to diagnose the three-dimensional circulation, including the vertical velocity, in the upper ocean from high-resolution observations of sea surface height and buoyancy. The formulation for the adiabatic component departs from the classical surface quasigeostrophic framework considered before since it takes into account the stratification within the surface mixed layer that is usually much weaker than that in the ocean interior. To achieve this, the model approximates the ocean with two constant stratification layers: a finite-thickness surface layer (or the mixed layer) and an infinitely deep interior layer. It is shown that the leading-order adiabatic circulation is entirely determined if both the surface streamfunction and buoyancy anomalies are considered. The surface layer further includes a diabatic dynamical contribution. Parameterization of diabatic vertical velocities is based on their restoring impacts of the thermal wind balance that is perturbed by turbulent vertical mixing of momentum and buoyancy. The model skill in reproducing the three-dimensional circulation in the upper ocean from surface data is checked against the output of a high-resolution primitive equation numerical simulation.
\end{abstract}

\section{Introduction}

Vertical velocities associated with mesoscale $O(100) \mathrm{km}$ and submesoscale $O(10) \mathrm{km}$ processes in the upper ocean are important for vertical exchanges of heat, salinity, and tracers, such as nutrients and dissolved organic and inorganic carbon, between the surface mixed layer (ML) and the ocean interior (Klein and Lapeyre 2009). These vertical velocities are usually weaker than $100 \mathrm{~m} \mathrm{day}^{-1}\left(1 \mathrm{~mm} \mathrm{~s}^{-1}\right)$ and are therefore very difficult to measure directly. Instead, their balanced components have often been estimated indirectly from observations of density and horizontal velocity using the omega equation (Hoskins et al. 1978), but such estimations require in situ observations obtained from highresolution ship surveys (e.g., Pollard and Regier 1992; Rudnick 1996; Legal et al. 2007). A decade ago, Lapeyre

Corresponding author address: Cédric Chavanne, Institut des Sciences de la Mer de Rimouski, Université du Québec à Rimouski, 310 allée des Ursulines, Rimouski, QC G5L 3A1, Canada. E-mail: cedric.chavanne@ensta.org and Klein (2006) and LaCasce and Mahadevan (2006) proposed a method to diagnose the three-dimensional (3D) balanced motions in the upper ocean from a single snapshot of surface buoyancy or height anomalies, combined with a knowledge of the background stratification, allowing to estimate vertical velocities in the upper ocean from satellite observations (Isern-Fontanet et al. 2006).

The method developed by Lapeyre and Klein (2006) is based on the principle of invertibility of potential vorticity (PV; Hoskins et al. 1985), which states that given a balance condition and a reference state, a knowledge of the global distribution of PV and of buoyancy at the boundaries is sufficient to diagnose all the other adiabatic dynamical fields, including vertical velocities. Using the fact that in the quasigeostrophic (QG) balance condition, PV is a linear operator, Lapeyre and Klein (2006) decomposed the PV problem into a surface problem (with a nonzero surface buoyancy and zero interior PV) and an interior problem (with zero surface buoyancy and nonzero interior PV). The total solution is then the sum of the surface and interior solutions. For the surface problem, 
the special case of an infinitely deep ocean with buoyancy anomalies vanishing at great depths has received much attention and was dubbed surface quasigeostrophic (SQG) dynamics by Held et al. (1995) (see also Blumen 1978).

Lapeyre and Klein (2006) showed that for a baroclinic unstable flow forced by a large-scale mean PV gradient including a nonzero surface buoyancy gradient, PV anomalies in the interior are correlated with surface buoyancy anomalies when large-scale buoyancy gradients at the surface and in the interior are correlated. As a result, the interior and surface solutions are correlated, and the total solution can be approximated in the upper ocean by an "effective" SQG (eSQG) solution, using a constant reference stratification $N(z)=N_{0}$ that must be determined empirically. While Lapeyre and Klein (2006) obtained spatial correlations greater than 0.85 between vertical velocities from a primitive equation $(\mathrm{PE}) \mathrm{nu}-$ merical simulation and those diagnosed by the eSQG inversion in the first $500 \mathrm{~m}$ of their model, Klein et al. (2009) obtained much poorer correlations $(<0.6)$ in the ML of another PE simulation with higher horizontal and vertical resolution, in which the ML was forced by spatially homogeneous high-frequency winds. Using the PE simulation of Klein et al. (2009), Ponte et al. (2013) showed that correlations for vertical velocities significantly increased at 40-m depth (within the ML) when adding diabatic vertical velocities due to turbulent vertical mixing of momentum (Garrett and Loder 1981) to the adiabatic vertical velocities diagnosed by the eSQG inversion.

However, the eSQG model, by considering a constant background stratification, does not take into account the fact that the stratification within the ML is usually much weaker than that in the ocean interior. The discontinuity between the ML and interior stratification leads to different physics for the ML than for the ocean interior by allowing interactions within the ML between edge waves associated with buoyancy anomalies at the ocean surface and ML base (Flierl et al. 2014). Such interactions lead to baroclinic instabilities as in the Eady problem (Eady 1949) that captures their main features in the QG approximation. These ML instabilities (MLIs; Stone 1971; Haine and Marshall 1998; Boccaletti et al. 2007; Fox-Kemper et al. 2008) have recently been shown to dominate vertical velocity variance in deep winter MLs in high-resolution numerical simulations of the northwestern Atlantic (Mensa et al. 2013) and North Pacific (Sasaki et al. 2014) Oceans. Observations in the western subtropical North Atlantic confirm this seasonal cycle in the ML submesoscale energy (Callies et al. 2015).

Two questions therefore arise: What are the relative contributions of adiabatic and diabatic dynamics to vertical velocities in the ML? Can vertical velocities associated with MLIs be diagnosed solely from surface observations? To address these questions, we develop the simplest possible diagnostic QG model that explicitly takes into account the presence of the ML in the ocean. We show that under the assumption of zero PV anomalies in the ML, buoyancy anomalies at the ML base can be recovered if observations of both surface buoyancy and height anomalies are taken into account. This enables a diagnosis, based solely on surface observations, of vertical velocities associated with MLIs in dynamical regimes where the Rossby number Ro $\ll$ 1. We also consider diabatic ML processes by including turbulent vertical mixing of momentum and buoyancy in the QG equations. We diagnose diabatic vertical velocities within the ML using a diabatic omega equation instead of the approximate solution of Garrett and Loder (1981) used by Ponte et al. (2013). The model, which we call MLQG hereinafter, is developed in section 2 and is then tested in section 3 against the numerical simulation reported in Klein et al. (2009) and Ponte et al. (2013). The originality and limitations of the MLQG model are discussed in section 4 . The main model equations are summarized in the appendix.

\section{Quasigeostrophic model with a surface mixed layer}

We start from the nondimensional primitive equations for hydrostatic and Boussinesq flow on the $f$ plane, neglecting turbulent horizontal mixing:

$$
\begin{aligned}
\operatorname{Ro} \frac{D^{*} u^{*}}{D^{*} t^{*}}-v^{*} & =-\frac{\partial p^{*}}{\partial x^{*}}+\operatorname{Ek} \frac{\partial}{\partial z^{*}}\left(A_{v}^{*} \frac{\partial u^{*}}{\partial z^{*}}\right) \\
\operatorname{Ro} \frac{D^{*} v^{*}}{D^{*} t^{*}}+u^{*} & =-\frac{\partial p^{*}}{\partial y^{*}}+\operatorname{Ek} \frac{\partial}{\partial z^{*}}\left(A_{v}^{*} \frac{\partial v^{*}}{\partial z^{*}}\right) \\
\frac{\partial p^{*}}{\partial z^{*}} & =b^{*} \\
\operatorname{Ro} \frac{D^{*} b^{*}}{D^{*} t^{*}}+\operatorname{Bu} N^{* 2} w^{*} & =\operatorname{Pr}^{-1} \operatorname{Ek} \frac{\partial}{\partial z^{*}}\left(K_{v}^{*} \frac{\partial b^{*}}{\partial z^{*}}\right) \\
\frac{\partial u^{*}}{\partial x^{*}}+\frac{\partial v^{*}}{\partial y^{*}}+\frac{\partial w^{*}}{\partial z^{*}} & =0
\end{aligned}
$$

where superscripts * indicate non-dimensional variables and $D^{*} / D^{*} t^{*}$ is the material derivative operator. The variables have been non-dimensionalized as follows: horizontal distance $(x, y)=L\left(x^{*}, y^{*}\right)$, vertical distance $z=H z^{*}$, horizontal velocity $(u, v)=U\left(u^{*}, v^{*}\right)$, vertical velocity $w=R o(U H / L) w^{*}$, where $R o=U / f_{0} L$ is the Rossby number and $f_{0}$ the (constant) Coriolis parameter, 
pressure $p=\rho_{0} f_{0} L U p^{*}$ ( $\rho_{0}$ is a typical value for seawater density), buoyancy $b=\left(f_{0} L U / H\right) b^{*}$, time $t=(L / U) t^{*}$, vertical turbulent viscosity $A_{v}=A_{0} A_{v}^{*}$, vertical turbulent diffusivity $K_{v}=K_{0} K_{v}^{*}$, and background buoyancy frequency $N(z)=N_{0} N^{*}(z)$. Ek $=A_{0} / f_{0} H^{2}$ is the Ekman number, $\mathrm{Bu}=\left(N_{0} H / f_{0} L\right)^{2}$ is the Burger number, and $\operatorname{Pr}=A_{0} / K_{0}$ is the Prandtl number. Boundary conditions are not written explicitly here, but they involve wind stress and air-sea heat and freshwater fluxes at the ocean surface. Turbulent vertical mixing is parameterized as Fickian fluxes, and countergradient buoyancy fluxes are not considered. Therefore, the model is not suitable for convective MLs.

We consider flow regimes for which $\mathrm{Ek}=O(\mathrm{Ro}) \ll 1$, $\mathrm{Bu}=O(1)$, and $\operatorname{Pr}$ is not too small so that $\operatorname{Pr}^{-1} \mathrm{Ek} \ll 1$. We decompose the flow variables into series of Rossby numbers:

$$
\begin{aligned}
\left(u^{*}, v^{*}, w^{*}, p^{*}, b^{*}\right)= & \left(u_{0}^{*}, v_{0}^{*}, w_{0}^{*}, p_{0}^{*}, b_{0}^{*}\right) \\
& +\operatorname{Ro}\left(u_{1}^{*}, v_{1}^{*}, w_{1}^{*}, p_{1}^{*}, b_{1}^{*}\right)+\text { H.O.T. },
\end{aligned}
$$

where H.O.T. refers to higher order terms. To the zeroth order in Ro, the flow is purely horizontal and in geostrophic balance:

$$
\begin{aligned}
& v_{0}^{*}=\frac{\partial p_{0}^{*}}{\partial x^{*}}, \\
& u_{0}^{*}=-\frac{\partial p_{0}^{*}}{\partial y^{*}}, \\
& b_{0}^{*}=\frac{\partial p_{0}^{*}}{\partial z^{*}}, \quad \text { and } \\
& w_{0}^{*}=0 .
\end{aligned}
$$

All flow variables at zeroth order in Ro can therefore be determined from the streamfunction $p_{0}^{*}$. To determine the latter, we use the principle of invertibility of QG PV:

$$
q_{0}^{*}=\Delta^{*} p_{0}^{*}+\mathrm{Bu}^{-1} \frac{\partial}{\partial z^{*}}\left(\frac{1}{N^{* 2}} \frac{\partial p_{0}^{*}}{\partial z^{*}}\right),
$$

where $\Delta^{*}$ is the horizontal Laplacian operator. Note that when diabatic and frictional processes are retained in the QG equations [Eqs. (1)-(5)], QG PV is not a conserved quantity. However, the principle of invertibility of PV holds whether or not diabatic and frictional processes are important (Hoskins et al. 1985).

To the zeroth order in Ro, the vertical velocity is zero. To diagnose vertical velocity, one must therefore consider the equations of motion at first order in Ro. Vertical velocities are diagnosed using the QG omega equation with turbulent vertical mixing terms (Nagai et al. 2006):

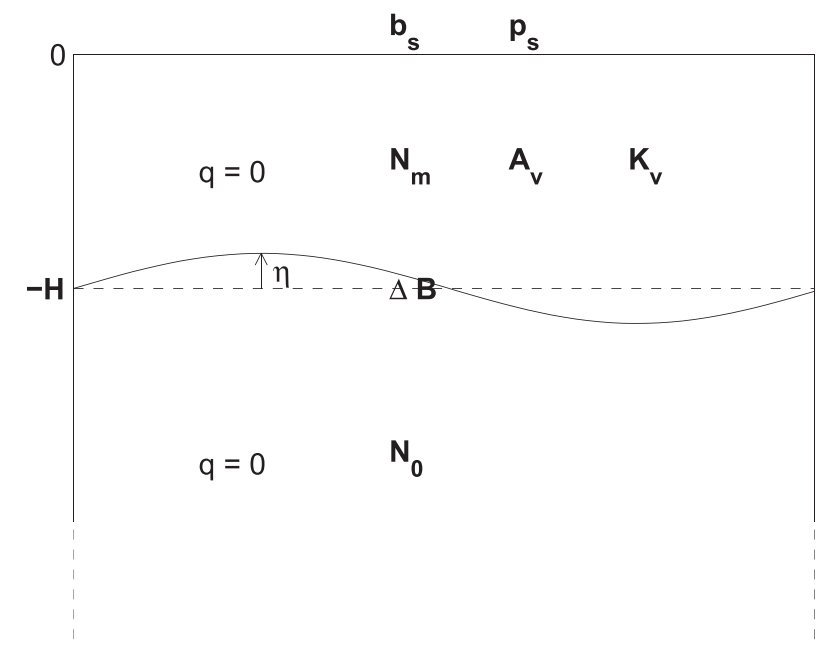

FIG. 1. Schematic of the MLQG model. Bold symbols represent the model inputs.

$$
\begin{aligned}
\mathrm{Bu} & N^{* 2} \Delta^{*} w_{1}^{*}+\frac{\partial^{2} w_{1}^{*}}{\partial z^{* 2}} \\
= & 2 \nabla^{*} \cdot \mathbf{Q}^{*}-\operatorname{Ro}^{-1} \mathrm{Ek}\left[\frac{\partial^{2}}{\partial z^{* 2}}\left(A_{v}^{*} \Delta^{*} b_{0}^{*}\right)\right. \\
& \left.-\operatorname{Pr}^{-1} \frac{\partial}{\partial z^{*}}\left(K_{v}^{*} \frac{\partial}{\partial z^{*}} \Delta^{*} b_{0}^{*}\right)\right]
\end{aligned}
$$

where $\nabla^{*}$ is the horizontal gradient operator, and $\mathbf{Q}^{*}=-\nabla^{*} \mathbf{u}_{0}^{*} \cdot \nabla^{*} b_{0}^{*}$ is the geostrophic $\mathbf{Q}$ vector (Hoskins et al. 1978).

We now proceed to determine the streamfunction $p_{0}^{*}$ analytically for the simplest possible QG model that takes into account the presence of the ML in the ocean (Fig. 1). We consider a surface ML of thickness $H$ at rest, with uniform stratification $N_{m}$ [i.e., $N^{*}\left(z^{*}\right)=N_{m} / N_{0}$ for $\left.-1<z^{*} \leq 0\right]$ and prescribed vertical turbulent viscosity and diffusivity profiles, overlying an infinitely deep adiabatic [i.e., $A_{v}^{*}\left(z^{*}\right)=K_{v}^{*}\left(z^{*}\right)=0$ for $z^{*}<-1$ ] interior layer with uniform stratification $N_{i}$ [i.e., $N^{*}\left(z^{*}\right)=N_{i} / N_{0}$ for $\left.z^{*}<-1\right]$. Here, layers refer to vertical domains with uniform stratification instead of uniform density as in traditional layered models. In addition, we allow for a buoyancy jump $\Delta B^{*}$ at the ML base typical of oceanic density profiles (e.g., Brainerd and Gregg 1995). Formally, the idealized stratification can be expressed as

$$
\begin{aligned}
N^{*}\left(z^{*}\right)= & \frac{N_{m}}{N_{0}} \mathcal{H}\left(z^{*}+1\right)+\Delta B^{* 1 / 2} \delta\left(z^{*}+1\right) \\
& +\frac{N_{i}}{N_{0}} \mathcal{H}\left(-z^{*}-1\right),
\end{aligned}
$$

where $\mathcal{H}$ is the Heaviside step function, and $\delta$ is the Dirac delta function. 

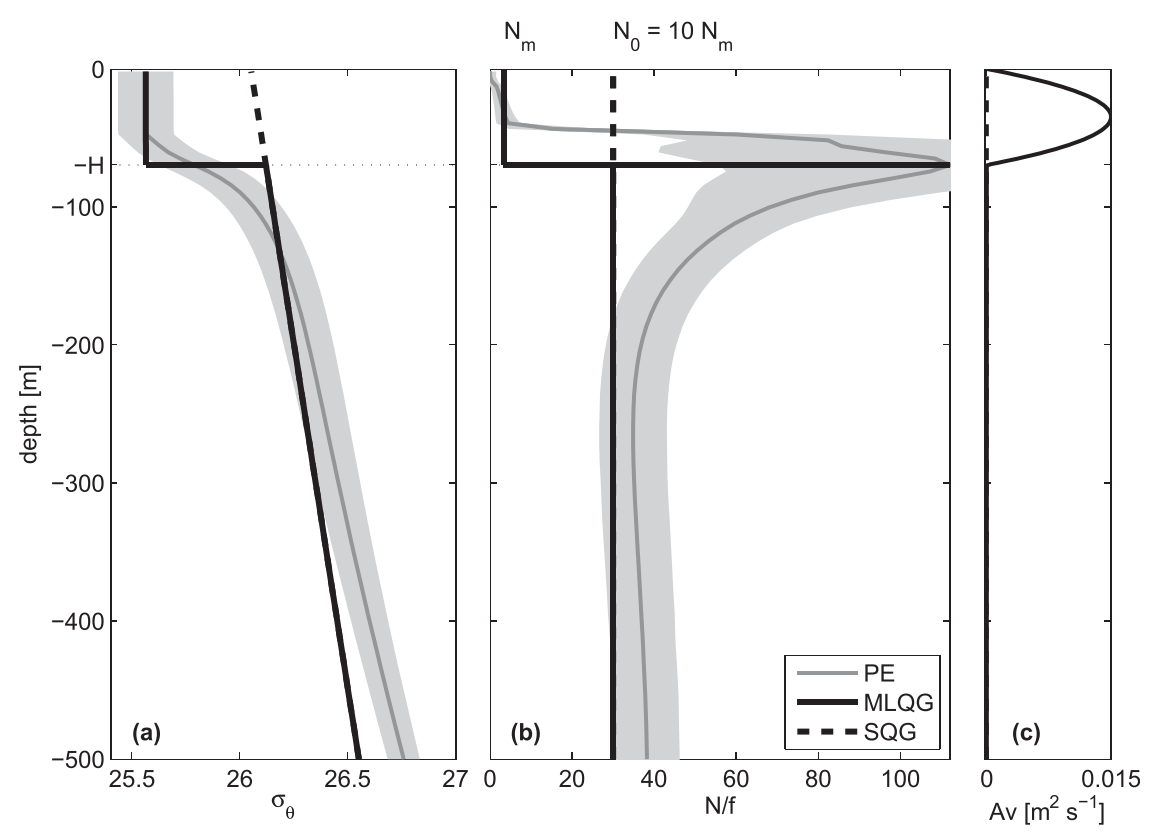

FIG. 2. (a) Potential density and (b) buoyancy frequency (normalized by $f$ ) in the PE simulation (gray line represents the horizontal average and shading represents the standard deviation) and in the MLQG (solid black line) and SQG (dashed black line) models. Dotted horizontal line represents the ML base in the MLQG model. Buoyancy $N_{0}$ in the MLQG and SQG models is lower than values of $N$ in the PE simulation between 100 and $500 \mathrm{~m}$ to take into account the effects of interior PV anomalies on the total solution [see text in introduction and Lapeyre and Klein (2006)]. (c) Turbulent vertical diffusivity prescribed in the MLQG model.

We follow the formalism of Lapeyre and Klein (2006) involving zero PV except at the boundaries, and therefore approximate the solution to Eq. (11) with inhomogeneous boundary conditions as the solution to

$$
\begin{aligned}
& \Delta^{*} p_{0}^{*}+\mathrm{Bu}_{m}^{-1} \frac{\partial^{2} p_{0}^{*}}{\partial z^{*^{2}}}=0 \text { for }-1<z^{*} \leq 0, \text { and } \\
& \Delta^{*} p_{0}^{*}+\mathrm{Bu}_{i}^{-1} \frac{\partial^{2} p_{0}^{*}}{\partial z^{*^{2}}}=0 \text { for } z^{*}<-1
\end{aligned}
$$

with inhomogeneous boundary conditions, where $\mathrm{Bu}_{m}=\left(N_{m} H / f_{0} L\right)^{2}$ is the ML Burger number, and $\mathrm{Bu}_{i}=$ $\left(N_{0} H / f_{0} L\right)^{2}$ is the interior Burger number. We have chosen to retain $N_{m}$ as the effective stratification in the ML, which amounts to neglecting the interior solution in the ML under the premise that QG PV is close to zero in the weakly stratified ML. In contrast, we use an effective stratification $N_{0} \neq N_{i}$ in the interior layer to approximate the effect of the interior solution on the total solution (Lapeyre and Klein 2006).

Boundary conditions must be specified to solve Eqs. (14) and (15). Neumann boundary conditions could be used by specifying buoyancy anomalies at the surface $\left(z^{*}=0\right)$ and the ML base $\left(z^{*}=-1\right)$ (Juckes 1994).
Here, with the aim of diagnosing the 3D circulation in the upper ocean solely from surface observations, we use instead both Neumann and Dirichlet boundary conditions at the surface by specifying surface buoyancy $b_{s}$ and surface streamfunction $p_{s}$. This uniquely determines the solution in the ML. To determine the solution in the interior, we use the matching condition at the ML base that involves the interface vertical displacement $\eta$ (Fig. 1):

$$
p^{*}\left(-1^{+}\right)-p^{*}\left(-1^{-}\right)=\Delta B^{*} \eta^{*}
$$

where $\eta^{*}=\eta / H=O(\mathrm{Ro})$. For a buoyancy jump $\Delta B=$ $5 \times 10^{-3} \mathrm{~m} \mathrm{~s}^{-2}$ [consistent with the stratification shown in Fig. 2 and observations of the density jump at the ML base reported, e.g., by Brainerd and Gregg (1995)], a ML depth anomaly of $20 \mathrm{~m}$ then only gives a streamfunction anomaly of $10^{3} \mathrm{~m}^{2} \mathrm{~s}^{-1}$, an order of magnitude smaller than the typical streamfunction values shown in Fig. 3 of $O\left(10^{4}\right) \mathrm{m}^{2} \mathrm{~s}^{-1}$. Therefore, to zeroth order in Ro, the streamfunction is continuous at the interface:

$$
p_{0}^{*}\left(-1^{+}\right)=p_{0}^{*}\left(-1^{-}\right)
$$

yielding a Dirichlet boundary condition at the interface for the interior solution. The remaining boundary 
condition is the Neumann condition that buoyancy anomalies vanish at great depths.

To solve Eqs. (14) and (15), subject to the boundary conditions just mentioned, we decompose the streamfunction in horizontal Fourier components: $p_{0}^{*}\left(x^{*}, y^{*}, z^{*}\right)=\hat{p}_{0}^{*}\left(k^{*}, l^{*}, z^{*}\right) \exp \left[i\left(k^{*} x^{*}+l^{*} y^{*}\right)\right]$,

where $k^{*}$ and $l^{*}$ are the zonal and meridional wavenumbers, respectively. The solution for the streamfunction is

$$
\hat{p}_{0}^{*}=\left\{\begin{array}{l}
\hat{p}_{s}^{*} \cosh \left(\mathrm{Bu}_{m}^{1 / 2} \kappa^{*} z^{*}\right)+\hat{b}_{s}^{*} \mathrm{Bu}_{m}^{-1 / 2} \kappa^{*-1} \sinh \left(\mathrm{Bu}_{m}^{1 / 2} \kappa^{*} z^{*}\right) \text { for }-1 \leq z^{*} \leq 0, \\
{\left[\hat{p}_{s}^{*} \cosh \left(\mathrm{Bu}_{m}^{1 / 2} \kappa^{*}\right)-\hat{b}_{s}^{*} \mathrm{Bu}_{m}^{-1 / 2} \kappa^{*-1} \sinh \left(\mathrm{Bu}_{m}^{1 / 2} \kappa^{*}\right)\right] \exp \left[\mathrm{Bu}_{i}^{1 / 2} \kappa^{*}\left(z^{*}+1\right)\right] \text { for } z^{*} \leq-1,}
\end{array}\right.
$$

where $\kappa^{*}=\left(k^{* 2}+l^{* 2}\right)^{1 / 2}$. Geostrophic velocities and buoyancy anomalies are then obtained using Eqs. (7)-(9):

$$
\begin{aligned}
& \hat{u}_{0}^{*}=-i l^{*} \hat{p}_{0}^{*}, \\
& \hat{v}_{0}^{*}=i k^{*} \hat{p}_{0}^{*}, \text { and } \\
& \hat{b}_{0}^{*}=\left\{\begin{array}{l}
\hat{p}_{s}^{*} \mathrm{Bu}_{m}^{1 / 2} \kappa^{*} \sinh \left(\mathrm{Bu}_{m}^{1 / 2} \kappa^{*} z^{*}\right)+\hat{b}_{s}^{*} \cosh \left(\mathrm{Bu}_{m}^{1 / 2} \kappa^{*} z^{*}\right) \text { for }-1<z^{*} \leq 0, \\
{\left[\hat{p}_{s}^{*} \mathrm{Bu}_{i}^{1 / 2} \kappa^{*} \cosh \left(\mathrm{Bu}_{m}^{1 / 2} \kappa^{*}\right)-\hat{b}_{s}^{*} \mathrm{Bu}_{m}^{-1 / 2} \mathrm{Bu}_{i}^{1 / 2} \sinh \left(\mathrm{Bu}_{m}^{1 / 2} \kappa^{*}\right)\right] \exp \left[\mathrm{Bu}_{i}^{1 / 2} \kappa^{*}\left(z^{*}+1\right)\right] \text { for } z^{*}<-1 .}
\end{array}\right.
\end{aligned}
$$

Buoyancy anomalies are discontinuous at the ML base due to the jump in background stratification [see Fig. 3 of Juckes (1994) for a simple illustration].

Since our parameterizations for vertical turbulent mixing are not suitable for convective MLs, we restrict our attention to stable conditions, for which $\operatorname{Pr}=1$ (Large et al. 1994). After developing the diabatic forcing terms in the omega equation [Eq. (12)], the omega equation for MLQG becomes

$$
\begin{aligned}
& \frac{\partial^{2} \hat{w}_{1}^{*}}{\partial z^{* 2}}-\mathrm{Bu} N^{* 2} \kappa^{* 2} \hat{w}_{1}^{*} \\
& \quad=2 \widehat{\nabla^{*} \cdot \mathbf{Q}^{*}}+\mathrm{Ro}^{-1} \mathrm{Ek}\left(\frac{d^{2} A_{v}^{*}}{d z^{* 2}} \kappa^{* 2} \widehat{b_{0}^{*}}+\mathrm{Bu} \frac{d A_{v}^{*}}{d z^{*}} \kappa^{* 4} \widehat{p_{0}^{*}}\right),
\end{aligned}
$$

with boundary conditions $\hat{w}_{1}^{*}\left(z^{*}=0\right)=\hat{w}_{1}^{*}\left(z^{*} \rightarrow-\infty\right)=0$.

To diagnose vertical velocities, we need to prescribe $A_{v}^{*}$. We chose a parabolic profile to yield nonzero values for both diabatic forcing terms on the right-hand side of Eq. (23), with $A_{v}^{*}=0$ at the surface and ML base because of the buoyancy jumps at these depths (Fig. 2c):

$$
A_{v}^{*}=-4 z^{*}\left(1+z^{*}\right) \text { for }-1 \leq z^{*} \leq 0 .
$$

In the next section, Eq. (23) is solved numerically using finite differences for each Fourier component. However, because of the Dirac delta function in $N^{*}$ [Eq. (13)], the ML depth must be treated differently. Vertically integrating Eq. (23) over an infinitesimally thin layer across the ML base yields

$$
\begin{gathered}
\left.\frac{\partial \hat{w}_{1}^{*}}{\partial z^{*}}\right|_{z^{*}=-1^{+}}-\left.\frac{\partial \hat{w}_{1}^{*}}{\partial z^{*}}\right|_{z^{*}=-1^{-}}-\mathrm{Bu} \Delta B^{*} \kappa^{* 2} \hat{w}_{1}^{*}(-1) \\
=\left.\operatorname{Ro}^{-1} \mathrm{Ek} \kappa^{* 2} \frac{d A_{v}^{*}}{d z^{*}}\right|_{z^{*}=-1^{+}} \widehat{b_{0}^{*}}\left(-1^{+}\right) .
\end{gathered}
$$

Equation (25) is discretized at $z^{*}=-1$ when numerically solving the omega equation [Eq. (23)].

The equations required to diagnose the $3 \mathrm{D}$ circulation in the upper ocean using the MLQG model developed in this section are recalled in the appendix in dimensional form.

\section{Numerical results}

\section{a. Numerical simulation}

We apply the MLQG model to data from a PE numerical simulation of a turbulent flow field in a zonal $\beta$-plane channel centered at $45^{\circ} \mathrm{N}$ with a size of $1000 \mathrm{~km}$ by $2000 \mathrm{~km}$ and a depth of $4000 \mathrm{~m}$. This numerical simulation has been described in detail by Klein et al. (2008, 2009), Danioux et al. (2011), and Ponte et al. (2013). Here, we only briefly describe its main features. The numerical code is the Regional Oceanic Modeling System (ROMS; Shchepetkin and McWilliams 2005), with the KPP parameterization for vertical mixing (Large et al. 1994) and a bi-Laplacian parameterization for horizontal mixing (with $A_{h}=2 \times 10^{6} \mathrm{~m}^{4} \mathrm{~s}^{-2}$ ). The domain is zonally periodic and has vertical walls at the northern and southern boundaries with free-slip boundary conditions. The spatial resolution is $2 \mathrm{~km}$ in 

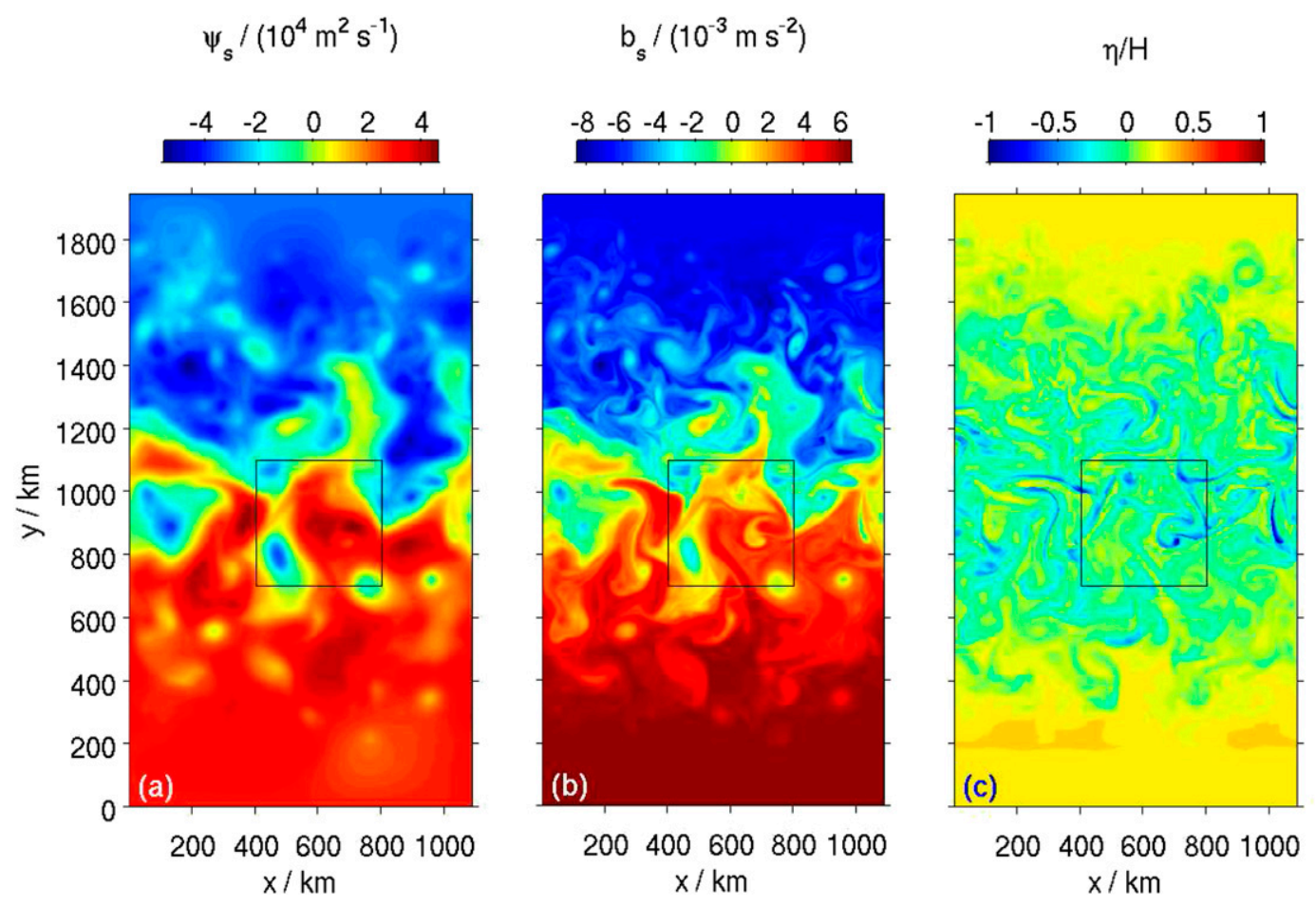

FIG. 3. (a) Streamfunction, (b) buoyancy anomalies, and (c) normalized interface displacements in the PE simulation. The black square delimits the area shown in Fig. 5.

the horizontal and 100 levels in the vertical (with vertical grid spacing ranging from $\sim 3 \mathrm{~m}$ near the surface to $\sim 200 \mathrm{~m}$ near the bottom). The horizontally averaged vertical profile of buoyancy frequency $N$ (Fig. 2b) features a permanent pycnocline centered at $600-\mathrm{m}$ depth (not shown) that corresponds to a first Rossby radius of deformation $R_{d}=23 \mathrm{~km}$. The mesoscale turbulence (Fig. 3) is forced by using a relaxation to a basic state that corresponds to a large-scale meridional density gradient that is surface intensified. An active ML with a mean depth of $\sim 60 \mathrm{~m}$ and a ML deformation radius $O(100) \mathrm{m}$, forced by spatially uniform but temporally varying winds and surface heat fluxes, caps a seasonal pycnocline. The data used here has been averaged over $18 \mathrm{~h}$ to filter out near-inertial oscillations.

\section{b. Diagnosis of balanced $3 D$ circulation in the upper ocean}

The resting depth of the interface between the two layers of the MLQG model was chosen to be the depth of maximum background buoyancy frequency of the PE simulation ( $H=70 \mathrm{~m}$; Fig. 2b). Background buoyancy frequencies for the MLQG model were chosen to be $N_{m} \sim 3 f_{0}$ and $N_{0} \sim 30 f_{0}$ (as in Klein et al. 2009) to approximate the vertical profile used in the PE simulation in the upper $500 \mathrm{~m}$. The value of $N_{0}$ is lower than the values of $N$ in the PE simulation between 100 and $500 \mathrm{~m}$ to take into account the effects of interior PV anomalies on the total solution (Lapeyre and Klein 2006), as discussed in the introduction. The buoyancy jump between the ML and the interior was chosen to be $\Delta B=5 \times 10^{-3} \mathrm{~m} \mathrm{~s}^{-2}$ to approximate the mean density profile of the PE simulation (Fig. 2a). The maximum vertical viscosity and diffusivity coefficients in the ML of the MLQG model were chosen to be $A_{0}=K_{0}=1.5 \times 10^{-2} \mathrm{~m}^{2} \mathrm{~s}^{-1}$ (Fig. $2 \mathrm{c}$ ), so that the ratio of the peak in MLQG vertical velocity variance in the ML over that in the interior matches that of the PE simulation (Fig. 4b). The corresponding Ekman depth is $h_{E}=\sqrt{2 A_{0} / f_{0}}=17 \mathrm{~m}$.

Figures $3 \mathrm{a}$ and $3 \mathrm{~b}$ show the surface streamfunction and buoyancy from the numerical simulation at an arbitrary time (after the simulation has reached statistical equilibrium). The background state (zonally averaged fields) was removed to obtain surface streamfunction and buoyancy anomalies. These 2D fields were Fourier transformed, and the 3D circulation was diagnosed with the MLQG model described in section 2. To assess the validity of the assumption behind the MLQG model, namely, that interface displacements are small compared to the ML depth [i.e., $\eta / H=(h-H) / H=O($ Ro $) \ll$ 1], the ML depth $h(x, y)$ was determined as the depth of the maximum value of buoyancy frequency at each grid point. Normalized interface displacements $\eta / H$ are shown in Fig. 3c. Although interface displacements can 

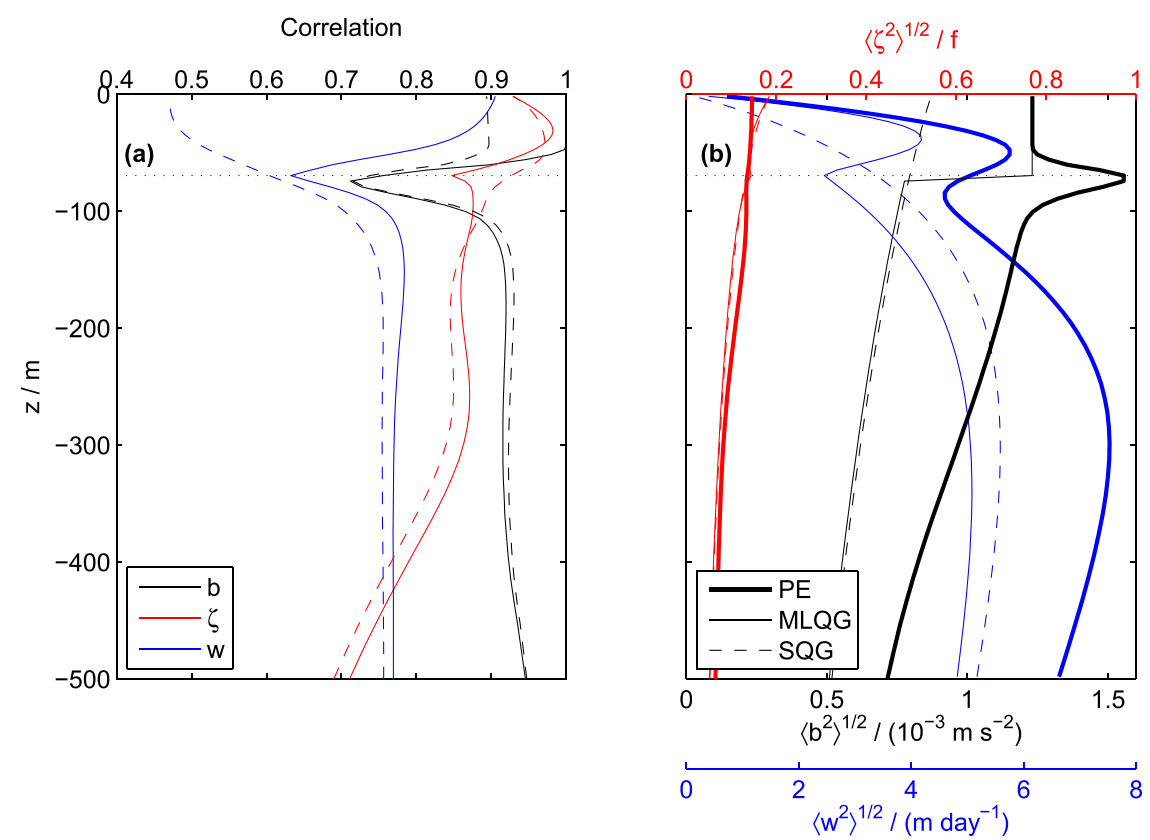

FIG. 4. (a) Spatial (horizontal) correlations as a function of depth between MLQG (solid lines) or SQG (dashed lines) and PE buoyancy anomalies (black), relative vorticities (red), and vertical velocities (blue). (b) Their standard deviations are shown for the PE simulation (thick solid lines) and MLQG (thin solid lines) and SQG (dashed lines) models. Horizontal dotted lines indicate the ML base in the MLQG model.

be as large as the average ML depth along some fronts, they remain less than $\sim 0.25 H$ over most of the domain. Therefore, the QG assumption behind the MLQG model seems reasonable, except at strong fronts.

Figure 4a shows spatial (horizontal) correlation coefficients $r$ as a function of depth between buoyancy anomalies, relative vorticities, and vertical velocities of the PE simulation and those diagnosed with the MLQG model. Relative vorticities are well correlated in the first $300 \mathrm{~m}(r>0.85)$, but the correlation decreases quickly below $300 \mathrm{~m}$. Buoyancy anomalies are almost perfectly correlated $(r \sim 1)$ in a large part of the ML because of the use of surface buoyancy anomalies as input to the MLQG model [Eq. (22)]. Correlation decreases to 0.7 at the interface between the two layers. The MLQG model is unable to reproduce the local enhancement of buoyancy anomalies in the seasonal pycnocline (Fig. 4b, black lines). Correlation increases again above 0.9 between 100 and $500 \mathrm{~m}$, but variance is underestimated. Vertical velocities are well correlated near the surface $(r \sim 0.9)$, in contrast to the SQG-diagnosed vertical velocities for which $r<0.5$ (dashed blue line in Fig. 4a), but correlation decreases to less than 0.7 (less than half the variance explained) in the seasonal pycnocline, below which it increases again to 0.77 between 100 and $500 \mathrm{~m}$. Even at the depth where the correlation is a minimum, the main patterns of vertical velocities are qualitatively well reproduced by the MLQG model (Fig. 5). The local enhancement of vertical velocity variance in the ML is also reproduced by the MLQG model but not by the SQG model (Fig. 4b, blue lines).

To understand the MLQG model strengths and limitations, it is helpful to look at correlation in the spectral domain or coherence $c$ (Fig. 6, bottom panels). For buoyancy anomalies (Fig. 6g), coherence is close to 1 at all horizontal scales in the ML because of the use of surface buoyancy anomalies as input to the MLQG model as mentioned above. However, coherence quickly drops in the seasonal pycnocline for wavelengths smaller than $100 \mathrm{~km}$, for which the validity of the QG approximation becomes questionable $\left(2 \pi R_{d}=145 \mathrm{~km}\right)$ but remains high for wavelengths between 100 and $350 \mathrm{~km}$. For relative vorticity (Fig. 6h), coherence is large at horizontal scales and depths where most of the energy resides (Fig. 6b), explaining the high spatial correlation coefficients above $300 \mathrm{~m}$. However, as horizontal scales decrease, the depth down to which coherence is large gets shallower, while energy remains fairly uniform with depth above $500 \mathrm{~m}$ in the PE simulation (Fig. 6b), explaining the decrease of the spatial correlation coefficient below 300-m depth (Fig. 4a). For vertical velocity (Fig. 6i), coherence is large at all wavelengths smaller than $350 \mathrm{~km}$ in the ML, and at wavelengths between 60 and $350 \mathrm{~km}$ below the ML, where most of the energy resides (Fig. 6c). 


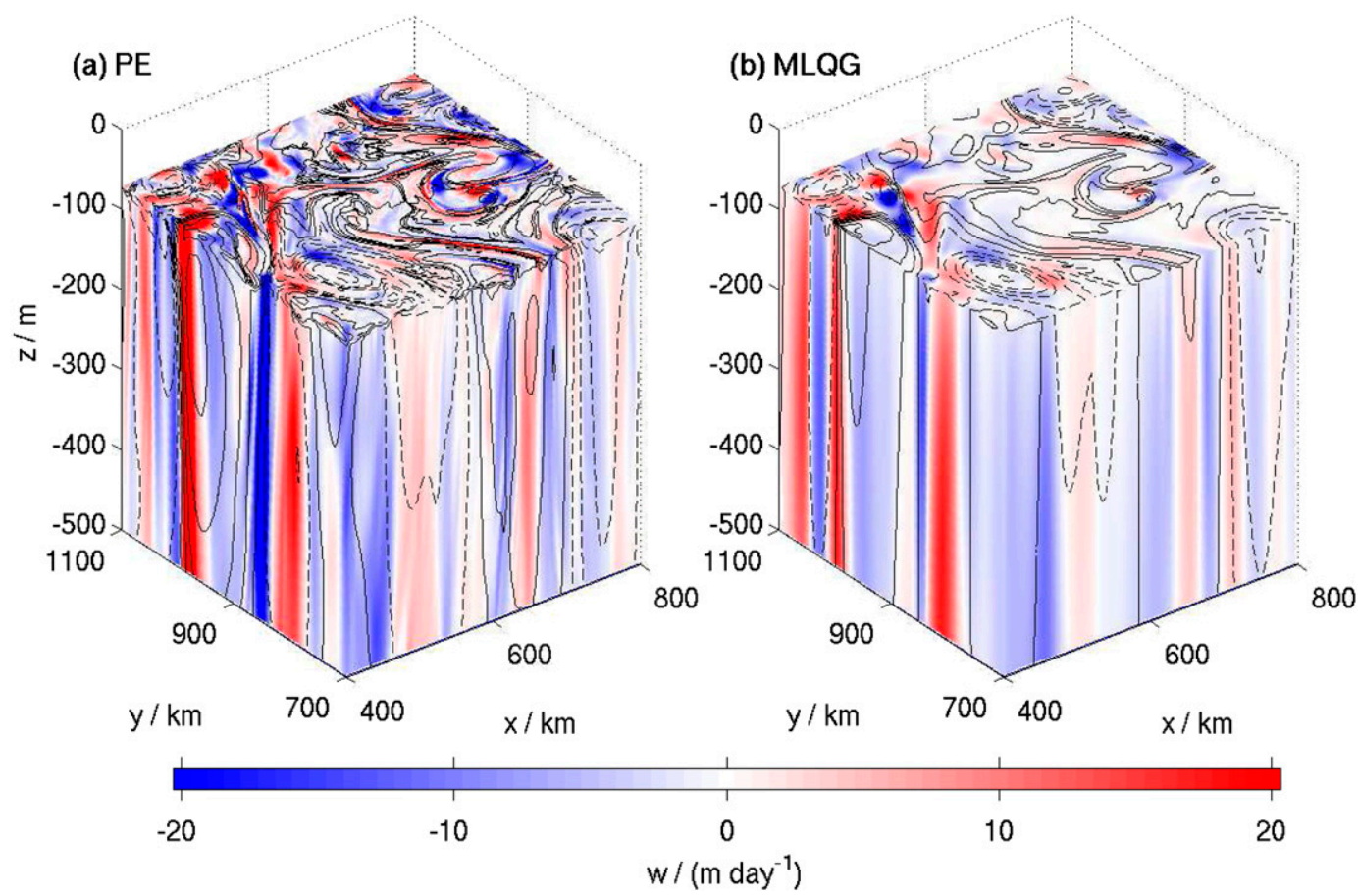

FIG. 5. Vertical and horizontal (at the ML base) sections of vertical velocities (color) and buoyancy anomalies (contours, spaced by $10^{-3} \mathrm{~m} \mathrm{~s}^{-2}$, solid for positive and dashed for negative values) are shown for (a) the PE simulation and (b) the MLQG model. The horizontal area is indicated by a black square in Fig. 3 .

To assess the relative contributions of adiabatic and diabatic dynamics to vertical velocities in the ML, the omega equation [Eq. (23)] is also solved by setting $A_{v}^{*}=0$ to obtain adiabatic vertical velocities $w_{a}^{*}$. Diabatic vertical velocities are then obtained as $w_{d}^{*}=w_{1}^{*}-w_{a}^{*}$ since the omega equation is linear in $w_{1}^{*}$. Figure 7 shows the relative contributions of $w_{a}^{*}$ and $w_{d}^{*}$ at 40-m depth in the ML. Adiabatic vertical velocities dominate the variance for wavelengths longer than $30 \mathrm{~km}$ and peak around $80 \mathrm{~km}$. They are well correlated with PE velocities $\left(c^{2}>0.6\right)$ for wavelengths between 50 and $350 \mathrm{~km}$. They are slightly better correlated with PE velocities than SQG-diagnosed vertical velocities are (cf. the blue and cyan curves in Fig. 7b), but the improvement is not sufficient to account for the much improved spatial correlations in the ML (Fig. 4a). These are due to the diabatic vertical velocities that dominate the variance at wavelengths shorter than $30 \mathrm{~km}$ and peak around $15 \mathrm{~km}$, at scales where PE vertical velocity variance is still high (black curve in Fig. 7a). Indeed, diabatic vertical velocities are well correlated with $\mathrm{PE}$ velocities for wavelengths shorter than $30 \mathrm{~km}$.

\section{Discussion}

A QG model with a ML (MLQG model) has been developed to diagnose the $3 \mathrm{D}$ circulation, including vertical velocities, in the upper ocean from only surface observations of buoyancy and height anomalies and knowledge of the background stratification. Diabatic dynamics (namely, turbulent vertical mixing) are included in the ML.

The formulation for the adiabatic component departs from the classical SQG framework since it takes into account the presence of the ML by considering two constant stratification layers: a finite-thickness weakly stratified ML and an infinitely deep interior layer. PV is assumed to be constant within each layer. So the solution for the adiabatic dynamical component within the ML is similar to the Eady solution and that in the interior to the SQG solution. This configuration allows us to diagnose vertical velocities associated with MLIs within the limitations of the QG approximation. As pointed out by Haine and Marshall (1998), although the QG analysis of Eady (1949) is formally inapplicable to the oceanic ML because of its finite Richardson number, it is yet qualitatively correct, and a similar qualitative success may be expected for the diagnostic model presented here. The main problems to be solved are the boundary conditions at the interface between the ML and the interior. Because the streamfunction is continuous at the ML base at zeroth order in Ro, knowledge of surface streamfunction and buoyancy anomalies are 


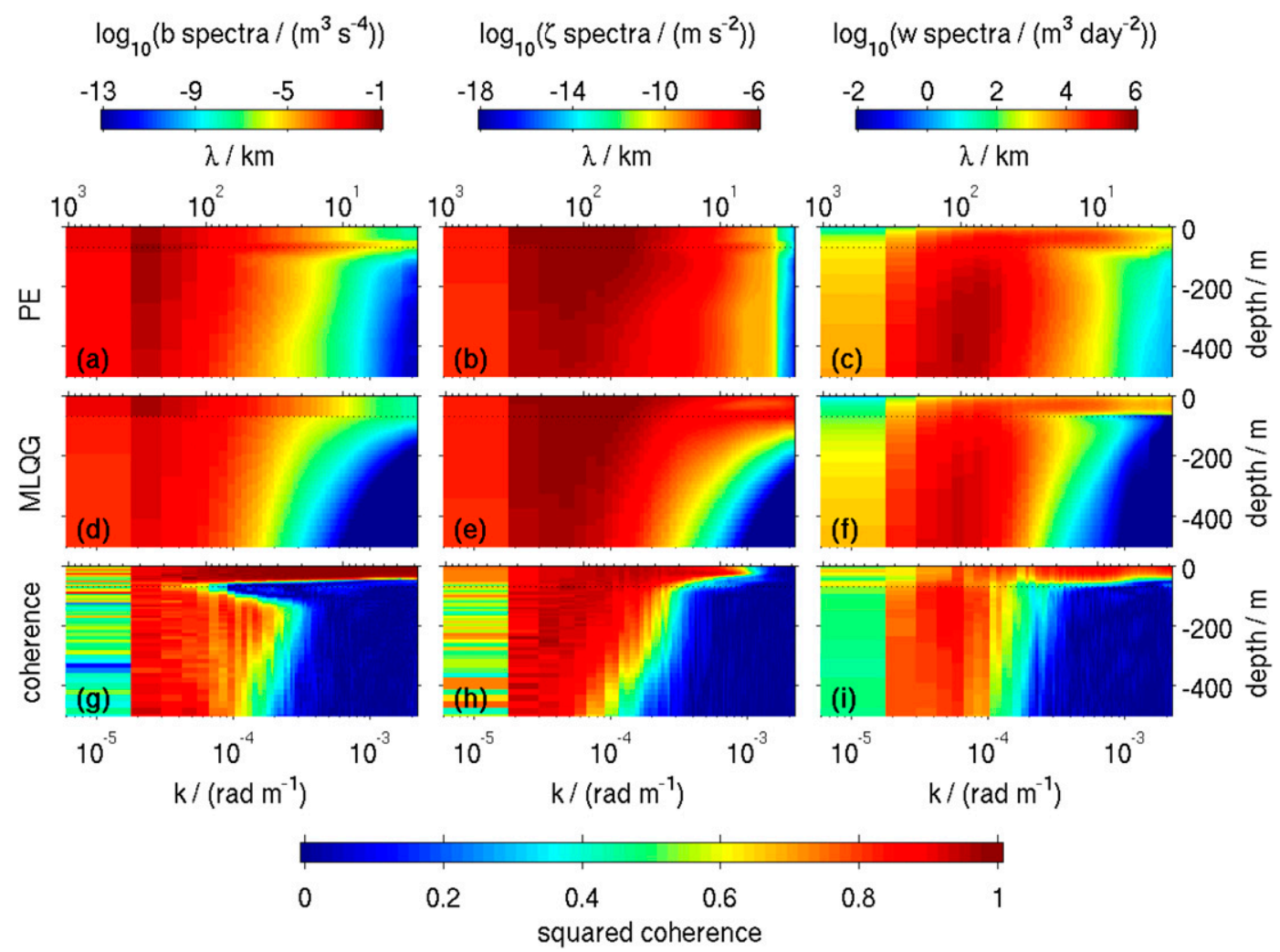

FIG. 6. Spectral distributions of (top) variance in the PE simulation, (middle) variance in the MLQG model, and (bottom) coherence between MLQG and PE for (left) buoyancy anomalies, (center) relative vorticities, and (right) vertical velocities. Dotted horizontal lines indicate the ML base in the MLQG model.

sufficient to determine the streamfunction at all depths, without requiring knowledge of buoyancy anomalies at the ML base. This is a key point of our model.

Recently, LaCasce and Wang (2015) also used a simplified QG model incorporating a surface ML to diagnose subsurface horizontal velocities and buoyancy anomalies from surface fields. Their model differs from ours in two aspects. First, they explicitly solve the interior problem by projecting interior PV anomalies on the first baroclinic mode. Second, they use a matching condition at the ML base not only for streamfunction (which they require to be continuous as we do), but also for buoyancy anomalies, so that the surface problem can be solved using only surface buoyancy anomalies. This leaves sea surface height, minus the contribution from the surface solution, to determine the interior solution. They tested two different matching conditions for buoyancy anomalies at the ML base: $b / N^{2}$, which guarantees continuity of $w$, and $b$ itself, which leads to a discontinuity in $w$, an embarrassing feature when attempting to diagnose vertical velocities as we do here. Although matching $b / N^{2}$ guarantees continuity of $w$ for adiabatic dynamics, it is not a necessary condition since what needs to be matched is actually $D\left(b / N^{2}\right) / D t$ [see Eq. (4)], which is done implicitly in our model through the omega equation.

The formulation for the diabatic component departs from that of Ponte et al. (2013), who used a 3D extension of the 2D analytical solution of Garrett and Loder (1981), which takes the nondimensional form

$$
w_{\mathrm{GL} 81}^{*}=-A_{v}^{*} \Delta^{*} b_{0}^{*} .
$$

To obtain their solution, Garrett and Loder (1981) implicitly assumed $\mathrm{Ro} \ll \mathrm{Ek} \ll 1$, a more restrictive assumption than that used to derive the omega equation [Eq. (12)], namely, Ro $=O(\mathrm{Ek}) \ll 1$. As a result, their solution is not the general solution to the diabatic omega equation [i.e., Eq. (12) with $\nabla^{*} \cdot \mathbf{Q}^{*}=0$ ]. However, in the limit of vanishing stratification in the ML (i.e., $\mathrm{Bu}_{m} \rightarrow 0$ ), buoyancy anomalies in the ML become depth independent [see Eq. (22)], and the solution to the diabatic omega equation asymptotes to the solution of Garrett and Loder (1981). With $N_{m} \sim 3 f_{0}$, the ML Rossby radius is only $\sim 200 \mathrm{~m}$, and so for wavelengths greater than $10 \mathrm{~km}$, $N_{m} \kappa H / f_{0} \lesssim 0.1$, and the limit of vanishing stratification in 


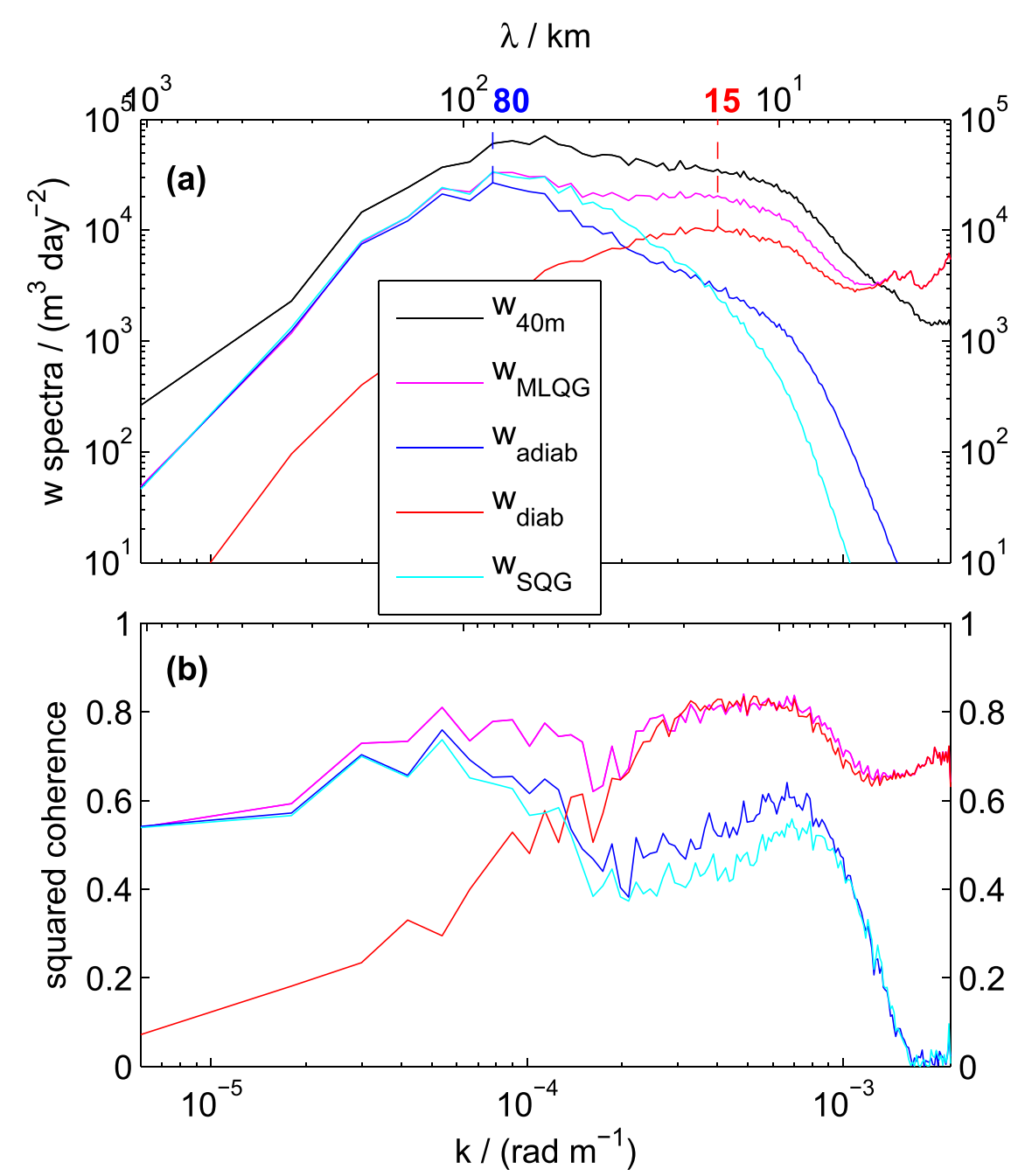

FIG. 7. (top) Spectra of vertical velocities at 40-m depth for the PE simulation (black) and the MLQG (magenta) and SQG (cyan) models. The adiabatic (blue) and diabatic (red) contributions to the MLQG vertical velocities are also shown. (bottom) Squared coherence between the diagnosed and PE vertical velocities.

the ML, and therefore the solution of Garrett and Loder (1981), becomes a good approximation. A peculiar feature of the diabatic omega equation is that when $\operatorname{Pr}=1$ and $A_{v}^{*}=K_{v}^{*}=1$ (i.e., vertical viscosity and diffusivity are depth independent in the ML), the diabatic forcing terms cancel each other, as noted by Nagai et al. (2006). Therefore, given boundary conditions $\hat{w}_{1}^{*}\left(z^{*}=0\right)=$ $\hat{w}_{1}^{*}\left(z^{*} \rightarrow-\infty\right)=0$, nonzero diabatic vertical velocities are obtained only for vertically varying viscosity and diffusivity profiles [see Eq. (23)].

The MLQG diagnosis was tested against highresolution PE simulations of a turbulent flow field in a zonal $\beta$-plane channel. The main improvement over the classical SQG diagnosis is obtained for vertical velocities in the ML, which are well correlated with the PE velocities at all scales (Figs. 6i, 7b). This improvement is due to the diabatic vertical velocities, whose magnitude is larger than that of the adiabatic vertical velocities for wavelengths shorter than $30 \mathrm{~km}$ (Fig. 7a). However, the ML depth in the PE simulation used here is moderate (60 $\mathrm{m})$, and consequently MLIs should be weak and are probably not even resolved with the 2-km horizontal resolution used here. For deeper MLs, such as in winter in large parts of the ocean, MLIs are much more energetic, and they dominate vertical velocity variance (see Fig. 5b of Sasaki et al. 2014). Whether the MLQG model would provide a good diagnostic for deep MLs remains to be verified.

There are many limitations to the MLQG model. One is the determination of the interior solution to the PV 
inversion problem. We have chosen to neglect the contribution of the interior solution in the ML under the premise that QG PV is close to zero in the weakly stratified ML. Below the ML, we have chosen to follow the approach of Lapeyre and Klein (2006) for its simplicity since the focus here was on the dynamics in the diabatic ML. More elaborate approaches have been proposed (e.g., Wang et al. 2013; Ponte and Klein 2013) and could possibly be adapted to the MLQG framework. Another limitation is the assumption of small Ek, which prevents resolving the surface Ekman layer. The effects of Ekman dynamics would be to modify the surface boundary condition for $w_{1}^{*}$. As discussed by Ponte et al. (2013), in the present PE simulation the QG vertical mixing terms dominate over nonlinear Ekman effects in driving ageostrophic motions. The neglect of Ekman dynamics is indeed supported by the high correlations obtained for vertical velocities in the ML. Perhaps the most important limitation is the assumption of small Ro. While the statistical estimation of Ro, $\langle\mathrm{Ro}\rangle=\left\langle\zeta^{2}\right\rangle^{1 / 2} / f$, is small $(\langle\mathrm{Ro}\rangle\langle 0.2$; Fig. $4 b)$ and the interface displacements are usually small compared to ML depth (Fig. 3c), local Ro $=|\zeta / f|$ and interface displacements can become large at strong fronts, where vertical velocities are expected to be high. Therefore, the MLQG model will underestimate vertical velocities at strong fronts. The QG approximation could be relaxed for a less restrictive approximation such as the semigeostrophic approximation (as in Badin 2013), but in the case of a model with two constant stratification layers such as MLQG, observations of buoyancy anomalies in the seasonal pycnocline would be required for the diagnosis because of the matching condition [(Eq. 16)].

Acknowledgments. CC thanks Glenn Flierl for a useful discussion on the matching condition [Eq. (16)]. We thank both reviewers who significantly contributed to the improvement of the original version of this work. This work was supported by an individual discovery grant to CC from the Natural Sciences and Engineering Research Council of Canada. Numerical simulations reported here were done at IDRIS (France) and on the Earth Simulator (Yokohama, Japan) through a memorandum of understanding signed between
IFREMER, CNRS, and JAMSTEC. PK acknowledges the support of IFREMER (through the MOU IFREMERJAMSTEC), CNRS (France), and the Agence Nationale pour la Recherche [Contracts ANR-05-CIGC-010, ANR-09-BLAN-0365-02 and ANR-10-LABX-19-01 (LabexMER)].

\section{APPENDIX}

\section{Dimensional MLQG Equations}

We recall here the main steps and equations required to diagnose the $3 \mathrm{D}$ circulation in the upper ocean from the sole knowledge of surface streamfunction $\psi_{s}$ and buoyancy $b_{s}$ anomalies [and background stratification $N(z)$ ] using the MLQG model developed in section 2. The equations are given in dimensional form for easier application to oceanographic data.

The first step consists of determining, from the background stratification $N(z)$, the depth $H$ of the ML base, the ML stratification $N_{m}$, the buoyancy jump at the ML base $\Delta B$, and the effective interior stratification $N_{0}$. Here, we chose the value $N_{0}=30 f_{0}$ used by Klein et al. (2009), but in general, $N_{0}$ can be determined using the procedure proposed by Lapeyre and Klein (2006). The MLQG background stratification is then given by

$$
N(z)=N_{m} \mathcal{H}(z+H)+\Delta B^{1 / 2} \delta(z+H)+N_{0} \mathcal{H}(-z-H),
$$

where $\mathcal{H}$ is the Heaviside step function, and $\delta$ is the Dirac delta function.

The second step consists of computing the 2D Fourier transforms $\hat{\psi}_{s}$ and $\hat{b}_{s}$. For the numerical simulation considered here, the fields are periodic in $x$ but not in $y$, so the domain was mirrored at the northern boundary to obtain fields periodic in both $x$ and $y$ and avoid spectral leakage. In general, the domain should be mirrored twice, first at the northern boundary, then at the eastern boundary of the first mirrored domain.

The third step consists of diagnosing the streamfunction and buoyancy anomalies. First, the streamfunction is obtained with

$$
\hat{\psi}_{0}= \begin{cases}\hat{\psi}_{s} \cosh \frac{N_{m} \kappa z}{f_{0}}+\hat{b}_{s} N_{m}^{-1} \kappa^{-1} \sinh \frac{N_{m} \kappa z}{f_{0}} & \text { for } \quad-H \leq z \leq 0, \\ \left(\hat{\psi}_{s} \cosh \frac{N_{m} \kappa H}{f_{0}}-\hat{b}_{s} N_{m}^{-1} \kappa^{-1} \sinh \frac{N_{m} \kappa H}{f_{0}}\right) \exp \frac{N_{0} \kappa(z+H)}{f_{0}} & \text { for } z \leq-H,\end{cases}
$$


where $\kappa=\left(k^{2}+l^{2}\right)^{1 / 2}$, and $k$ and $l$ are the zonal and meridional wavenumbers, respectively. Second, the horizontal velocities and buoyancy anomalies are obtained with

$$
\begin{aligned}
& \hat{u}_{0}=-i l \hat{\psi}_{0}, \\
& \hat{v}_{0}=i k \hat{\psi}_{0}, \text { and }
\end{aligned}
$$

$$
\hat{b}_{0}= \begin{cases}\hat{\psi}_{s} N_{m} \kappa \sinh \frac{N_{m} \kappa z}{f_{0}}+\hat{b}_{s} \cosh \frac{N_{m} \kappa z}{f_{0}} & \text { for } \quad-H<z \leq 0, \\ \left(\hat{\psi}_{s} N_{0} \kappa \cosh \frac{N_{m} \kappa H}{f_{0}}-\hat{b}_{s} N_{0} N_{m}^{-1} \sinh \frac{N_{m} \kappa H}{f_{0}}\right) \exp \frac{N_{0} \kappa(z+H)}{f_{0}} & \text { for } \quad z<-H .\end{cases}
$$

The fourth and final step consists of diagnosing vertical velocities using the omega equation:

$$
\frac{\partial^{2} \hat{w}_{1}}{\partial z^{2}}-\left(\frac{N \kappa}{f}\right)^{2} \hat{w}_{1}=\frac{2}{f^{2}} \widehat{\nabla \cdot \mathbf{Q}}+\frac{d^{2} A_{v}}{d z^{2}} \frac{\kappa^{2}}{f^{2}} \widehat{b}_{0}+\frac{d A_{v}}{d z} \frac{N^{2} \kappa^{4}}{f^{3}} \widehat{\psi}_{0},
$$

where $\nabla$ is the horizontal gradient operator, and $\mathbf{Q}=$ $-\nabla \mathbf{u}_{0} \cdot \nabla b_{0}$. Boundary conditions are $\hat{w}_{1}(z=0)=$ $\hat{w}_{1}(z \rightarrow-\infty)=0$. To compute $\mathbf{Q}$, the spatial velocity and buoyancy fields must be obtained by taking the inverse 2D Fourier transforms of $\hat{\mathbf{u}}_{0}=\left(\hat{u}_{0}, \hat{v}_{0}\right)$ and $\hat{b}_{0}$ and restricting them to the original nonmirrored domain before computing their horizontal gradients.

The term $A_{v}$ is prescribed as a parabolic profile to yield nonzero values for both diabatic forcing terms on the right-hand side of Eq. (A6), with $A_{v}=0$ at the surface and ML base because of the buoyancy jumps at these depths (Fig. 2c):

$A_{v}=-4 A_{0} \frac{z}{H}\left(1+\frac{z}{H}\right)$ for $\quad-H \leq z \leq 0$,

where $A_{0}$ is the maximum vertical turbulent viscosity value.

Equation (A6) is solved numerically using finite differences for each Fourier component. However, because of the Dirac delta function in $N$ [Eq. (A1)], the ML depth must be treated differently. Vertically integrating Eq. (A6) over an infinitesimally thin layer across the ML base yields

$$
\begin{aligned}
& \left.\frac{\partial \hat{w}_{1}}{\partial z}\right|_{z=-H^{+}}-\left.\frac{\partial \hat{w}_{1}}{\partial z}\right|_{z=-H^{-}}-\Delta B \frac{\kappa^{2}}{f^{2}} \hat{w}_{1}(-H) \\
& =\left.\frac{\kappa^{2}}{f^{2}} \frac{d A_{v}}{d z}\right|_{z=-H^{+}} \widehat{b}_{0}\left(-H^{+}\right) .
\end{aligned}
$$

Equation (A8) is discretized at $z=-H$ when numerically solving the omega equation [Eq. (A6)].

\section{REFERENCES}

Badin, G., 2013: Surface semi-geostrophic dynamics in the ocean. Geophys. Astrophys. Fluid Dyn., 107, 526-540, doi:10.1080/ 03091929.2012.740479.

Blumen, W., 1978: Uniform potential vorticity flow: Part I. Theory of wave interactions and two-dimensional turbulence. J. Atmos. Sci., 35, 774-783, doi:10.1175/1520-0469(1978)035<0774: UPVFPI $>2.0 . \mathrm{CO} ; 2$.

Boccaletti, G., R. Ferrari, and B. Fox-Kemper, 2007: Mixed layer instabilities and restratification. J. Phys. Oceanogr., 37, 22282250, doi:10.1175/JPO3101.1.

Brainerd, K. E., and M. C. Gregg, 1995: Surface mixed and mixing layer depths. Deep-Sea Res. I, 42, 1521-1543, doi:10.1016/ 0967-0637(95)00068-H.

Callies, J., R. Ferrari, J. M. Klymak, and J. Gula, 2015: Seasonality in submesoscale turbulence. Nat. Commun., 6, 6862, doi:10.1038/ ncomms7862.

Danioux, E., P. Klein, M. W. Hecht, N. Komori, G. Roullet, and S. Le Gentil, 2011: Emergence of wind-driven near-inertial waves in the deep ocean triggered by small-scale eddy vorticity structures. J. Phys. Oceanogr., 41, 1297-1307, doi:10.1175/ 2011JPO4537.1.

Eady, E. T., 1949: Long waves and cyclone waves. Tellus, 1A, 3352, doi:10.1111/j.2153-3490.1949.tb01265.x.

Flierl, G., R. Ferrari, B. Fox-Kemper, and J. Callies, 2014: MultiEady models of surface and deep instabilities. 2014 Fall Meeting, San Francisco, CA, Amer. Geophys. Union, Abstract OS33E-01.

Fox-Kemper, B., R. Ferrari, and R. Hallberg, 2008: Parameterization of mixed layer eddies. Part I: Theory and diagnosis. J. Phys. Oceanogr., 38, 1145-1165, doi:10.1175/ 2007JPO3792.1.

Garrett, C. J. R., and J. W. Loder, 1981: Dynamical aspects of shallow sea fronts. Philos. Trans. Roy. Soc. London, B302, 563-581, doi:10.1098/rsta.1981.0183.

Haine, T. W., and J. Marshall, 1998: Gravitational, symmetric, and baroclinic instability of the ocean mixed layer. J. Phys. Oceanogr., 28, 634-658, doi:10.1175/1520-0485(1998)028<0634: GSABIO $>2.0 . \mathrm{CO} ; 2$.

Held, I. M., R. T. Pierrehumbert, S. T. Garner, and K. L. Swanson, 1995: Surface quasi-geostrophic dynamics. J. Fluid Mech., 282, 1-20, doi:10.1017/S0022112095000012.

Hoskins, B. J., I. Draghici, and H. C. Davies, 1978: A new look at the $\omega$-equation. Quart. J. Roy. Meteor. Soc., 104, 31-38, doi:10.1002/qj.49710443903.

—, M. E. McIntyre, and A. W. Robertson, 1985: On the use and significance of isentropic potential vorticity maps. Quart. J. Roy. Meteor. Soc., 111, 877-946, doi:10.1002/qj.49711147002. 
Isern-Fontanet, J., B. Chapron, G. Lapeyre, and P. Klein, 2006: Potential use of microwave sea surface temperatures for the estimation of ocean currents. Geophys. Res. Lett., 33, L24608, doi:10.1029/2006GL027801.

Juckes, M., 1994: Quasigeostrophic dynamics of the tropopause. J. Atmos. Sci., 51, 2756-2768, doi:10.1175/1520-0469(1994)051<2756: QDOTT>2.0.CO;2.

Klein, P., and G. Lapeyre, 2009: The oceanic vertical pump induced by mesoscale and submesoscale turbulence. Annu. Rev. Mar. Sci., 1, 351-375, doi:10.1146/annurev.marine.010908.163704.

_ B. B. Hua, G. Lapeyre, X. Capet, S. Le Gentil, and H. Sasaki, 2008: Upper ocean turbulence from high-resolution 3D simulations. J. Phys. Oceanogr., 38, 1748-1763, doi:10.1175/ 2007JPO3773.1.

—, J. Isern-Fontanet, G. Lapeyre, G. Roullet, E. Danioux, B. Chapron, S. Le Gentil, and H. Sasaki, 2009: Diagnosis of vertical velocities in the upper ocean from high resolution sea surface height. Geophys. Res. Lett., 36, L12603, doi:10.1029/ 2009 GL038359.

LaCasce, J. H., and A. Mahadevan, 2006: Estimating subsurface horizontal and vertical velocities from sea surface temperature. J. Mar. Res., 64, 695-721, doi:10.1357/002224006779367267.

_ surface fields with idealized stratification. J. Phys. Oceanogr., 45, 2424-2435, doi:10.1175/JPO-D-14-0206.1.

Lapeyre, G., and P. Klein, 2006: Dynamics of the upper oceanic layers in terms of surface quasigeostrophy theory. J. Phys. Oceanogr., 36, 165-176, doi:10.1175/JPO2840.1.

Large, W. G., J. C. McWilliams, and S. C. Doney, 1994: Oceanic vertical mixing: A review and a model with a nonlocal boundary layer parameterization. Rev. Geophys., 32, 363-403, doi:10.1029/94RG01872.

Legal, C., P. Klein, and A.-M. Treguier, 2007: Diagnosis of the vertical motions in a mesoscale stirring region. J. Phys. Oceanogr., 37, 1413-1424, doi:10.1175/JPO3053.1.
Mensa, J. A., Z. Garraffo, A. Griffa, T. M. Özgökmen, A. Haza, and M. Veneziani, 2013: Seasonality of the submesoscale dynamics in the Gulf Stream region. Ocean Dyn., 63, 923-941, doi:10.1007/s10236-013-0633-1.

Nagai, T., A. Tandon, and D. L. Rudnick, 2006: Two-dimensional ageostrophic secondary circulation at ocean fronts due to vertical mixing and large-scale deformation. J. Geophys. Res., 111, C09038, doi:10.1029/2005JC002964.

Pollard, R. T., and L. A. Regier, 1992: Vorticity and vertical circulation at an ocean front. J. Phys. Oceanogr., 22, 609-625, doi:10.1175/1520-0485(1992)022<0609:VAVCAA > 2.0.CO;2.

Ponte, A. L., and P. Klein, 2013: Reconstruction of the upper ocean 3D dynamics from high-resolution sea surface height. Ocean Dyn., 63, 777-791, doi:10.1007/s10236-013-0611-7.

,-- X. Capet, P.-Y. Le Traon, B. Chapron, and P. Lherminier, 2013: Diagnosing surface mixed layer dynamics from highresolution satellite observations: Numerical insights. J. Phys. Oceanogr., 43, 1345-1355, doi:10.1175/JPO-D-12-0136.1.

Rudnick, D. L., 1996: Intensive surveys of the Azores front: 2. Inferring the geostrophic and vertical velocity fields. J. Geophys. Res., 101, 16 291-16303, doi:10.1029/96JC01144.

Sasaki, H., P. Klein, B. Qiu, and Y. Sasai, 2014: Impact of oceanic-scale interactions on the seasonal modulation of ocean dynamics by the atmosphere. Nat. Commun., 5, 5636, doi:10.1038/ncomms6636.

Shchepetkin, A. F., and J. C. McWilliams, 2005: The Regional Ocean Modeling System: A split-explicit, free-surface, topography following coordinates ocean model. Ocean Modell., 9, 347-404, doi:10.1016/j.ocemod.2004.08.002.

Stone, P. H., 1971: Baroclinic stability under non-hydrostatic conditions. J. Fluid Mech., 45, 659-671, doi:10.1017/S0022112071000260.

Wang, J., G. R. Flierl, J. H. LaCasce, J. L. McClean, and A. Mahadevan, 2013: Reconstructing the ocean's interior from surface data. J. Phys. Oceanogr., 43, 1611-1626, doi:10.1175/ JPO-D-12-0204.1. 